\title{
BRUGADA TYPE ECG PATTERN IN A CASE OF ALUMINIUM PHOSPHIDE POISONING
}

Devinder Singh Mahajan, Jang Bahadur Singh, Rajeev Kumar Chaudhry

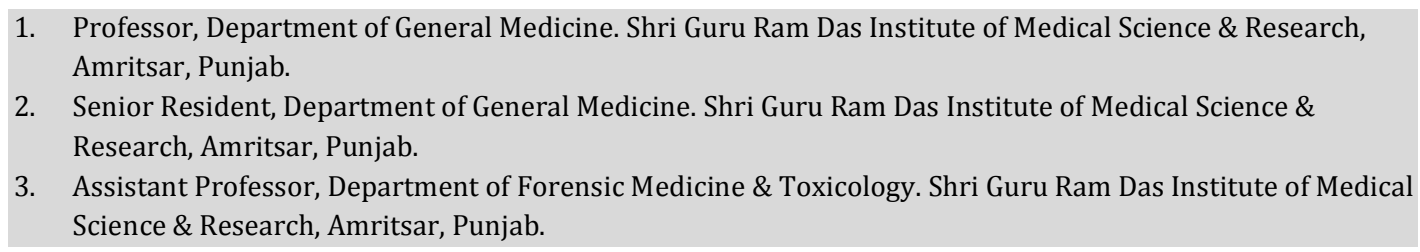

\section{CORRESPONDING AUTHOR}

Dr. Devinder Singh Mahajan, House No. 2, Guru Nanak Avenue, Majitha Road, Amritsar - 143004. E-mail: drdevin2008@yahoo.com Ph: 0091 98885-14390

ABSTRACT:_We are presenting a case which reveals unmasking of Brugada pattern with aluminum phosphide (celphos) ingestion that could most probably be due to hypomagnesaemia caused by the chemical, though its direct toxic effect on cardiac tissue cannot be ruled out. Earlier low levels of magnesium has been shown to precipitate arrhythmias in susceptible individuals and low magnesium levels have been recorded in survivors of sudden death with underlying Brugada syndrome, this is the first case in which celphos induced hypomagnesaemia has unmasked the Brugada pattern in ECG that can increase susceptibility to life threatening arrhythmias. This case opens scope for further research on role of magnesium in celphos poisoning.

KEY WORDS: Brugada Pattern, celphos, hypomagesaemia, cardiac tissue, arrhythmias.

A CASE REPORT:_A 20 years old male presented in the emergency department one hour after ingestion of two tablets of Celphos. He vomited 5 times before admission and was brought in a state of shock and altered sensorium. He did not complain of chest pain, palpitation or breathlessness. His pulse was 145/min, irregularly irregular with pulse deficit of 15 and poor volume. Systolic blood pressure was $80 \mathrm{mmHg}$ and respiratory rate was18/min. His systemic examination did not reveal any positive finding except drowsiness. His routine blood investigations were within normal limits except serum magnesium level of $1.0 \mathrm{mmol} / \mathrm{lt}$ and serum potassium of $2.2 \mathrm{mEq} / \mathrm{lt}$. ECG showed typical (type-I phenotype) of Brugada Syndrome with atrial fibrillation (AF) with heart rate of $\sim 145 / \mathrm{min}$. (Fig-1). Gastric lavage was done and patient was given I/V fluids with ionotropic support, I/V magnesium \& potassium supplements and hydrocortisone. Patient's condition stabilized with treatment and next day serum electrolytes normalized except serum magnesium which still measured $1.2 \mathrm{mmol} / \mathrm{lt}$ \& ECG showed persistence of the same pattern. ECHO showed normal cardiac functions. On day 4, serum magnesium level was $2.0 \mathrm{mmol} / \mathrm{lt}$ and ECG showed type III Brugada pattern (decreased ST elevation) with regular heart rate of 107/min (Fig--2). ECGs of his mother and brother were normal, however, the provocative tests and genetic testing were declined. Patient had to be discharged on persistent requests. 
DISCUSSION: Brugada syndrome described by Brugada \& Brugada in 1992 is a disease presenting with ST segment elevation in right precordial leads $V_{1}-V_{3}$ (in absence of acute coronary syndrome), right bundle branch block, susceptibility to VT (ventricular tachycardia) and structurally normal heart. It is common in apparently healthy young Southeast Asians with male to female ratio of 8:1. Clinical manifestations include syncope or cardiac arrest because of sudden arrhythmia, the most typical being rapid polymorphic VT that can degenerate into ventricular fibrillation and may culminate into sudden death.

Familial distribution consistent with autosomal dominant inheritance has also been reported with genetic mutations involving SCN5A gene in $20-25 \%$ of cases and GPD1-L in $~ 1 \%$ of cases.

The intermittent nature of ECG pattern complicates its diagnosis. 3 types of ECG patterns have been identified, namely type I (coved type ST segment elevation in precordial leads $V_{1}, V_{2}$ and $V_{3}$ ), type II (saddle-back type) and type 3 (right precordial saddle back type ST segment with mirror deviation from the isoelectric segment) ${ }^{1}$. Out of these "coved" ST segment elevation or type 1 is presently considered diagnostic for Brugada syndrome. ${ }^{2}$

Since in the present case there is no history of syncope or arrhythmias, no family h/o sudden death and moreover, no genetic or electrophysiological inducibility study could be done, this patient with type 1 ECG pattern of Brugada may be categorized as having Idiopathic Brugada ECG pattern. ${ }^{3}$

The underlying cellular mechanism of syndrome involves loss of dome of action potential because of disequilibrium between $\mathrm{I}_{\mathrm{to}}$ and $\mathrm{I}_{\mathrm{ca}}$ during phase 1 of action potential in right ventricular epicardium resulting in ST segment elevation. ${ }^{4}$ This heterogenous loss of action potential dome leads to its propagation from sites where it is maintained to sites where it is lost resulting in ventricular arrhythmias. ${ }^{5}$ Interventions increasing potassium current will increase ST segment elevation while interventions increasing calcium current will decrease ST elevation. Magnesium blocks the cellular efflux of potassium through $\mathrm{K}^{+}$channels in cardiomyocytes and also acts as a cofactor of NaKATPase. ${ }^{6}$ Thus, hypomagnesaemia which was present in the case at onset could be the cause of unmasking of Brugada pattern and is corroborated by the persistence of pattern till serum magnesium level normalized. Other electrolytes (including potassium) normalized within a day, thus, could not have been the causation factors.

The Brugada pattern was present in the absence of any provocative drugs like Nachannel blockers and class $1_{\mathrm{c}}$ antiarrhythmics like ajmaline, procainamide or flecainide which are known to unmask the concealed forms of syndrome. ${ }^{2}$

Other conditions that can simulate this pattern include electrolyte disturbances like hyperkalemia and hypercalcemia ${ }^{7}$ which were ruled out by normal serum calcium and low s. potassium at onset. Hypokalemia which was present at onset is probably not the cause of unmasking of pattern since it causes prolongation of AP duration in contrast to what occurs in Brugada syndrome though it may precipitate arrhythmias in susceptible individuals.

The effect of temperature change on ECG, both hypothermia ${ }^{4}$ (which simulates the pattern by J-point elevation) and fever ${ }^{8}$ (which can precipitate arrhythmia in rare cases of Brugada syndrome) have been ruled out by normal temperature records at presentation and during follow up.

Autonomic nervous system modulation can modify the phenotype because I/V administration of isoproterenol attenuates and that of acetylcholine accentuates the ECG abnormalities in affected individuals. ${ }^{2}$ This patient required ionotropic support for one day but 
the phenotype could not have been affected by that since the pattern persisted even after tapering the drugs.

Bradycardia has been known to increase ST segment elevation in the pt with Brugada syndrome and tachycardia to decrease the same. ${ }^{9}$ But this is reverse in our patient since tachycardia (heart rate-136/mt) at presentation was accompanied by Brugada pattern which gradually regressed with stabilization of heart rate.

Various studies on ECG abnormalities in aluminum phosphide poisoning have been conducted earlier but no such Brugada type pattern or unmasking of the phenotype in a patient with Brugada syndrome have ever been recorded. Mathur reported sinus tachycardia in $26.6 \%$ cases and complete heart block in $13.33 \%$ cases $^{10}$. In another study Chugh has reported conduction disturbances, ischemic changes, early repolarisation, varied sinoatrial blocks, bradycardia-tachycardia syndrome and electrical alternans in cases of celphos poisoning. This is probably the first case where the Brugada type pattern has been observed in celphos poisoning.

\section{REFERENCES:}

1. Wilde AAM ---- Spontaneous electrocardiographic fluctuations in Brugada Syndrome: does it matter? Eur Heart J. 2006;27(21):2493-4.

2. Priori SG, Napolitano C, Schwartz PJ ---- Genetics of cardiac arrhythmias. In: Libby P, Bonow RO, Mann DL, Zipes DP editors ---- Braunwald's Heart Disease: A Textbook of Cardiovascular Medicine, $8^{\text {th }}$ ed. Philadelphia: Saunders, 2008: 101-110.

3. Wilde AAM, Antzelevitch C, Borggrefe M, Brugada J, Brugada P, Brugada R, et al ----Proposed diagnostic criteria for the Brugada Syndrome: Consensus report. Circulation. 2002;106:2514-19.

4. Brugada Syndrome ---- cellular mechanisms (available at http://www.brugada.org/about/disease-cellularmechanisms. html accessed on 14-82008.

5. Olgin JE, Zipes DP ---- Specific Arrhythmias: Diagnosis and Treatment. In: Libby P, Bonow RO, Mann DL, Zipes DP editors ---- Braunwald's Heart Disease: A Textbook of Cardiovascular Medicine, 8th ed. Philadelphia: Saunders, 2008: 863-922.

6. Pansin P, Wathanavaha A, Tosukhowong P, Sriboonlue P, Tungsanga K, Sitrija V ---Magnesium and zinc status in survivors of sudden unexplained death syndrome in North East Thailand. Southeast Asian J Trop Med Public Health. 2002;33(1):172-8.

7. Mirivis DM, Goldberger AL ---- Electrocardiography. In: Libby P, Bonow RO, Mann DL, Zipes DP editors ---- Braunwald's Heart Disease: A Textbook of Cardiovascular Medicine, $8^{\text {th }}$ ed. Philadelphia: Saunders, 2008: 149-194.

8. Keller DI, Huang H, Zhao J, Frank R, Suarez V, Brink M, et al ---- A novel SCN5A mutation, F1344S, identified in a patient with Brugada Syndrome and fever-induced VF. Cardiovascular Research. 2006;70(3):521-29.

9. Brugada Syndrome ---- Effect of changes in Heart Rate and Exercise. (available at http://www.brugada.org/about/disease-chngsinheartratexercise accessed on 14-82008.

10. Mathur A, Swaroop A, Agarwal A. ECG changes in acute organophosphorus and aluminium phosphide poisoning. The Indian Practitioner 1999;52(4):249-52.

11. Chugh SN, Chugh K, Ram S, Malhotra KC. Electrocardiographic abnormalities in aluminium phosphide poisoning with special reference to its incidence, pathogenesis, mortality and histopathology. J Indian Med Assoc. 1991;89(2):32-5 
CASE REPORT
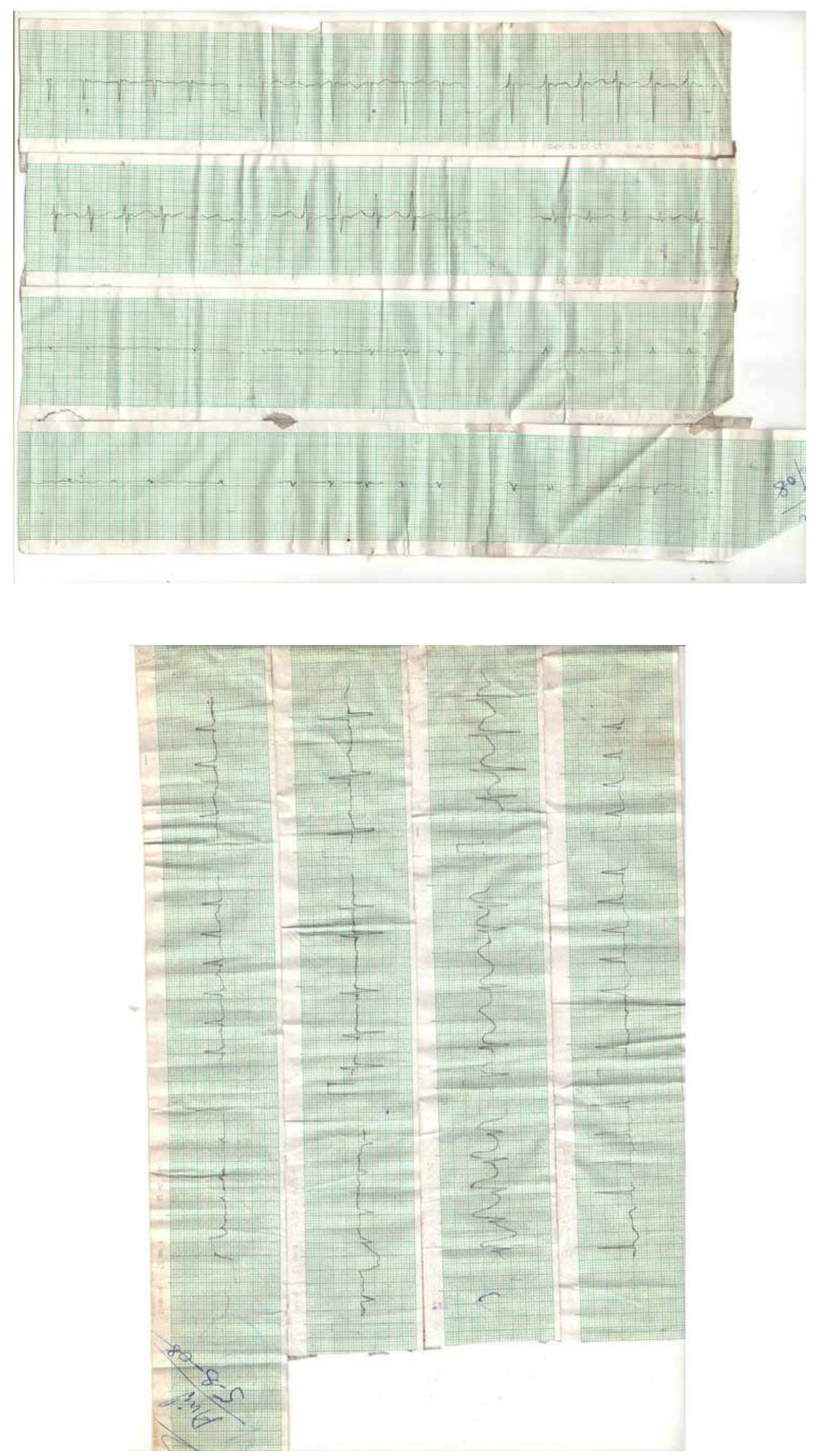